\title{
COMMENTARY Breaking down fear memory (Commentary on Meins et al.)
}

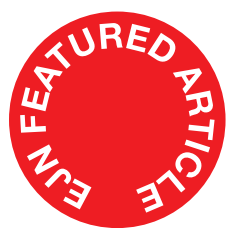

\author{
Stephen Maren \\ Department of Psychology and Neuroscience Program, University of Michigan, Ann Arbor, MI, USA
}

It is essential to rapidly learn and unfailingly remember threats in the environment. It is equally important to learn when those threats have passed, as well as the unique contexts in which one is safe from threat. In recent years, considerable progress has been made in understanding the neural circuits and molecular mechanisms that underlie the acquisition of fear memory in the mammalian brain (LeDoux, 2000; Maren, 2001). However, much less is known concerning the mechanisms for fear extinction, the learning process that suppresses fear when past threats no longer yield aversive outcomes.

Early work on the neural mechanisms of fear extinction revealed an essential role for $N$-methyl-D-aspartate (NMDA) receptors in the basolateral amygdala in fear extinction (Falls et al., 1992). More recently, the extinction circuit has expanded to include a network of inhibitory neurons in the amygdala, the intercalated cell masses (ITC), which play an important role in limiting the activity of neurons in the central nucleus (CE) that drive fear responses (Likhtik et al., 2008; Amano et al., 2010). In the current issue, Meins et al. (2010) shed new light on the molecular mechanisms within these inhibitory amygdala circuits that are involved in the extinction of fear. Using a molecular genetic approach in mice, they first show that inhibitory interneurons in the CE and ITC express a serine protease inhibitor, protease-nexin 1 (PN-1), which has previously been shown to regulate NMDA receptor function (Kvajo et al., 2004). Much weaker PN-1 expression was found in the basolateral nucleus of the amygdala (BA). Given the localization of PN-1 to inhibitory neurons in ITC and CE, Meins and colleagues next examined fear conditioning and extinction in PN-1 knockout mice. Interestingly, PN-1 knockouts exhibited normal fear conditioning, but had marked impairments in the extinction of conditional fear. Coupled with these behavioral deficits in extinction, PN-1 knockout mice exhibited reduced Fos expression in BA, as well as a reduction in phosphorylated alpha-calcium-calmodulin protein kinase II ( $\alpha$ CamKII) in ITC neurons after extinction training. Hence, these data reveal an important and novel role for PN-1 activity in extinction learning, and reinforce the important role for inhibitory interneurons in the amygdala in this process.

It has been proposed that NMDA receptor-dependent plasticity in the ITC is a mechanism for extinction learning (Amano et al., 2010). Insofar as PN-1 knockout mice exhibit impaired NMDA receptor function, the reduction of ITC c-Fos expression and $\alpha$ CamKII phosphorylation is consistent with this possibility. Nonetheless, recent data indicate that NMDA receptor antagonism in the CE (and presumably ITC) does not affect the acquisition of extinction in rats (Zimmerman \& Maren, 2010). Further work is clearly required to understand the precise role for amygdaloid NMDA receptors and PN-1 regulation of NMDA receptor function in fear extinction. Nonetheless, the work by Meins and colleagues reveals a new player in the molecular organization of extinction learning within inhibitory interneurons of the amygdala, a finding that yields exciting new avenues for research in this rapidly moving field.

\section{References}

Amano, T., Unal, C.T. \& Pare, D. (2010) Synaptic correlates of fear extinction in the amygdala. Nat. Neurosci., 13, 489-494.

Falls, W.A., Miserendino, M.J. \& Davis, M. (1992) Extinction of fear-potentiated startle: blockade by infusion of an NMDA antagonist into the amygdala. J. Neurosci., 12, 854-863.

Kvajo, M., Albrecht, H., Meins, M., Hengst, U., Troncoso, E., Lefort, S., Kiss, J.Z., Petersen, C.C.H. \& Monard, D. (2004) Regulation of brain proteolytic activity is necessary for the in vivo function of NMDA receptors. J. Neurosci., 24, 9734-9743.

LeDoux, J.E. (2000) Emotion circuits in the brain. Annu. Rev. Neurosci., 23, 155-184.

Likhtik, E., Popa, D., Apergis-Schoute, J., Fidacaro, G.A. \& Pare, D. (2008) Amygdala intercalated neurons are required for expression of fear extinction. Nature, 454, 642-645.

Maren, S. (2001) Neurobiology of Pavlovian fear conditioning. Annu. Rev. Neurosci., 24, 897-931.

Meins, M., Herry, C., Muller, C., Ciocchi, S., Moreno, E., Luthi, A. \& Monard, D. (2010) Impaired fear extinction in mice lacking protease nexin-1. Eur. J. Neurosci., 31, 2033-2042.

Zimmerman, J.M. \& Maren, S. (2010) NMDA receptor antagonism in the basolateral but not central amygdala blocks the extinction of Pavlovian fear conditioning in rats. Eur. J. Neurosci., 31, 1664-1670. 